\title{
Research Article \\ Products of Composition and Differentiation between the Fractional Cauchy Spaces and the Bloch-Type Spaces
}

\author{
R. A. Hibschweiler \\ Department of Mathematics and Statistics, University of New Hampshire, 03824 Durham, New Hampshire, USA
}

Correspondence should be addressed to R. A. Hibschweiler; rita.hibschweiler@unh.edu

Received 12 March 2021; Accepted 23 April 2021; Published 10 May 2021

Academic Editor: Gangadharan Murugusundaramoorthy

Copyright $\odot 2021$ R. A. Hibschweiler. This is an open access article distributed under the Creative Commons Attribution License, which permits unrestricted use, distribution, and reproduction in any medium, provided the original work is properly cited.

The operators $D C_{\Phi}$ and $C_{\Phi} D$ are defined by $D C_{\Phi}(f)=(f \circ \Phi)^{\prime}$ and $C_{\Phi} D(f)=f^{\prime} \circ \Phi$ where $\Phi$ is an analytic self-map of the unit disc and $f$ is analytic in the disc. A characterization is provided for boundedness and compactness of the products of composition and differentiation from the spaces of fractional Cauchy transforms $F_{\alpha}$ to the Bloch-type spaces $B^{\beta}$, where $\alpha>0$ and $\beta>0$. In the case $\beta<2$, the operator $D C_{\Phi}: F_{\alpha} \longrightarrow B^{\beta}$ is compact $\Leftrightarrow D C_{\Phi}: F_{\alpha} \longrightarrow B^{\beta}$ is bounded $\Leftrightarrow \Phi^{\prime} \in B^{\beta}, \Phi \Phi^{\prime} \in B^{\beta}$ and $\|\Phi\|_{\infty}<1$. For $\beta<1, C_{\Phi} D: F_{\alpha} \longrightarrow B^{\beta}$ is compact $\Leftrightarrow C_{\Phi} D: F_{\alpha} \longrightarrow B^{\beta}$ is bounded $\Leftrightarrow \Phi \in B^{\beta}$ and $\|\Phi\|_{\infty}<1$.

\section{Introduction}

Let $U=\{z \in C:|z|<1\}$ and let $H(U)$ denote the family of functions analytic on $U$. Let $M$ denote the Banach space of complex Borel measures on $T=\{x \in C:|x|=1\}$, endowed with the total variation norm. For $\alpha>0$, the space $F_{\alpha}$ of fractional Cauchy transforms is the family of functions of the form

$$
f(z)=\int_{T} \frac{1}{(1-\bar{x} z)^{\alpha}} d \mu(x)(|z|<1)
$$

where $\mu \in M$. The principal branch of the logarithm is used here. The space $F_{\alpha}$ is a Banach space, with norm

$$
\|f\|_{F_{\alpha}}=\inf \|\mu\|
$$

where $\mu$ varies over all measures in $M$ for which (1) holds. The families $F_{\alpha}$ have been studied extensively $[1,2]$. Interest in these spaces was first established in connection with the classical family $S$ of normalized univalent functions. It is known that $S \subseteq F_{\alpha}$ for any $\alpha>2$ [2]. The reference [2] also includes MacGregor's construction of a function $f \in S$ with $f \notin F_{2}$.
Let $\beta>0$. The Bloch-type space $B^{\beta}$ is the Banach space of functions analytic in $U$ such that $\sup _{z \in U}\left(1-|z|^{2}\right)^{\beta}\left|f^{\prime}(z)\right|$ $<\infty$, with norm

$$
\|f\|_{B^{\beta}}=|f(0)|+\sup _{z \in U}\left(1-|z|^{2}\right)^{\beta}\left|f^{\prime}(z)\right| .
$$

The relation (1) implies that $F_{\alpha} \subset B^{\alpha+1}$, and there is a constant $C$ depending only on $\alpha$ such that $\|f\|_{B^{\alpha+1}} \leq C\|f\|_{F_{\alpha}}$ for all $f \in F_{\alpha}$.

Let $\Phi$ be an analytic self-map of $U$. The composition operator $C_{\Phi}$ is defined by $C_{\Phi}(f)=f \circ \Phi$ for $f \in H(U)$. The differentiation operator $D$ is defined by $D(f)=f^{\prime}$. In this paper, the products $C_{\Phi} D(f)=f^{\prime} \circ \Phi$ and $D C_{\Phi}(f)=\Phi^{\prime}\left(f^{\prime} \circ \Phi\right)$ are studied. Conditions on $\Phi$ are given, necessary and sufficient to imply boundedness or compactness of $C_{\Phi} D: F_{\alpha} \longrightarrow B^{\beta}$ and $D C_{\Phi}: F_{\alpha} \longrightarrow B^{\beta}$.

Products of composition and differentiation on the Bloch space were studied by Ohno in [3]. In [4], Li and Stević studied $C_{\Phi} D$ and $D C_{\Phi}$ acting between the weighted Bergman spaces and the Bloch-type spaces. In [5], Hibschweiler and Portnoy studied these operators between Bergman and Hardy spaces. 


\section{Preliminary Results}

Fix $\alpha>0$. For fixed $z \in U$ and for $n=0,1, \ldots$, the relation (1) yields a constant $C$ depending only on $n$ such that $\left|f^{(n)}(z)\right|$ $\leq C\|f\|_{F_{\alpha}} /\left(1-|z|^{2}\right)^{\alpha+n}[2]$.

For each $w \in U,\left\|1 /(1-\bar{w} z)^{\alpha}\right\|_{F_{\alpha}}=1[2]$.

We follow the convention that $C$ denotes a positive constant, the precise value of which will differ from one appearance to the next.

Lemma 1 and Lemma 2 will be used to develop test functions for $F_{\alpha}$. Proofs appear in [6].

Lemma 1. Fix $\alpha>0$. For $w \in U$, define

$$
h_{w}(z)=\frac{1-|w|^{2}}{(1-\bar{w} z)^{\alpha+1}}(z \in U) \text {. }
$$

Then, $h_{w} \in F_{\alpha}$, and there is a constant $C$ such that $\left\|h_{w}\right\|_{F_{\alpha}} \leq C$ for all $w \in U$.

Lemma 2. Fix $\alpha>0$. For $w \in U$, define

$$
k_{w}(z)=\frac{\left(1-|w|^{2}\right)^{2}}{(1-\bar{w} z)^{\alpha+2}}(z \in U) .
$$

Then, $k_{w} \in F_{\alpha}$, and there is a constant $C$ such that $\left\|k_{w}\right\|_{F_{\alpha}} \leq C$ for all $w \in U$.

\section{The Operator $D C_{\Phi}: F_{\alpha} \longrightarrow B^{\beta}$}

In [7], Shapiro proved that the condition $\|\Phi\|_{\infty}<1$ is necessary for $C_{\Phi}: X \longrightarrow X$ to be compact, for Banach spaces $X$ obeying boundary regularity and Möbius invariance. In particular, Shapiro's result applies to the Lipschitz spaces and thus, to the space $B^{\gamma}$ when $\gamma<1$ [8].

Theorem 3. Fix $\alpha>0$ and $0<\beta<2$. Let $\Phi$ be an analytic selfmap of $U$.

$$
\begin{aligned}
& D C_{\Phi}: F_{\alpha} \longrightarrow B^{\beta} \text { is bounded } \Leftrightarrow \\
& \Phi^{\prime} \in B^{\beta}, \Phi \Phi^{\prime} \in B^{\beta} \text { and }\|\Phi\|_{\infty}<1 \Leftrightarrow \\
& D C_{\Phi}: F_{\alpha} \longrightarrow B^{\beta} \text { is compact. }
\end{aligned}
$$

Proof. First, assume that $D C_{\Phi}: F_{\alpha} \longrightarrow B^{\beta}$ is bounded, that is, there is a constant $C$ such that $\left\|D C_{\Phi}(f)\right\|_{B^{\beta}} \leq C\|f\|_{F_{\alpha}}$ for all $f \in F_{\alpha}$. It is clear that $\Phi^{\prime}=D C_{\Phi}(z) \in B^{\beta}$ and $\Phi \Phi^{\prime}=D C_{\Phi}\left(z^{2}\right.$ 12) $\in B^{\beta}$. Thus,

$$
\left(1-|z|^{2}\right)^{\beta}\left|\Phi^{\prime \prime}(z)\right| \leq C,
$$

and

$$
\left(1-|z|^{2}\right)^{\beta}\left|\Phi(z) \Phi^{\prime \prime}(z)+\left(\Phi^{\prime}(z)\right)^{2}\right| \leq C
$$

for all $z \in U$. It follows that

$$
\sup _{z \in U}\left(1-|z|^{2}\right)^{\beta}\left|\Phi^{\prime}(z)\right|^{2}<\infty
$$

and thus, $\Phi \in B^{\beta / 2}$.

Let $w \in U$ and define

$$
g_{w}(z)=\frac{\alpha+1}{\left(1-\Phi \overline{(w) z)^{\alpha}}\right.}-\frac{\alpha\left(1-|\Phi(w)|^{2}\right)}{\left(1-\Phi \overline{(w) z)^{\alpha+1}}\right.}(z \in U)
$$

By Lemma 1 and the preliminary results, there is a constant $C$ independent of $w$ such that $\left\|g_{w}\right\|_{F_{\alpha}} \leq C$, and thus, $\left\|D C_{\Phi}\left(g_{w}\right)\right\|_{B^{\beta}}=\left\|\left(g_{w}{ }^{\prime} \circ \Phi\right) \Phi^{\prime}\right\|_{B^{\beta}} \leq C$. It follows that

$\sup _{z \in U}\left(1-|z|^{2}\right)^{\beta}\left|g_{w}{ }^{\prime \prime}(\Phi(z))\left(\Phi^{\prime}(z)\right)^{2}+g_{w}{ }^{\prime}(\Phi(z)) \Phi^{\prime \prime}(z)\right| \leq C$,

for all $w \in U$. Calculations yield $g_{w}{ }^{\prime}(\Phi(w))=0$ and

$$
g_{w}^{\prime \prime}(\Phi(w))=\frac{-\alpha(\alpha+1) \Phi \overline{(w)^{2}}}{\left(1-|\Phi(w)|^{2}\right)^{\alpha+2}}
$$

The substitution $z=w$ in (11) now yields

$$
\sup _{w \in U}\left(1-|w|^{2}\right)^{\beta} \frac{\alpha(\alpha+1)|\Phi(w)|^{2}\left|\Phi^{\prime}(w)\right|^{2}}{\left(1-|\Phi(w)|^{2}\right)^{\alpha+2}} \leq C,
$$

and thus,

$$
\sup _{|\Phi(w)|>1 / 2} \frac{\left(1-|w|^{2}\right)^{\beta}\left|\Phi^{\prime}(w)\right|^{2}}{\left(1-|\Phi(w)|^{2}\right)^{\alpha+2}}<\infty
$$

By the relation (9),

$$
\sup _{|\Phi(z)| \leq 1 / 2} \frac{\left(1-|w|^{2}\right)^{\beta}\left|\Phi^{\prime}(w)\right|^{2}}{\left(1-|\Phi(w)|^{2}\right)^{\alpha+2}}<\infty
$$

Thus,

$$
C=\sup _{w \in U} \frac{\left(1-|w|^{2}\right)^{\beta}\left|\Phi^{\prime}(w)\right|^{2}}{\left(1-|\Phi(w)|^{2}\right)^{\alpha+2}}<\infty
$$

It follows that

$$
\sup _{w \in U} \frac{\left(1-|w|^{2}\right)^{\beta / 2}\left|\Phi^{\prime}(w)\right|}{\left(1-|\Phi(w)|^{2}\right)^{\beta / 2}} \leq \sup _{w \in U} \frac{\left(1-|w|^{2}\right)^{\beta / 2}\left|\Phi^{\prime}(w)\right|}{\left(1-|\Phi(w)|^{2}\right)^{(\alpha+2) / 2}}<\infty
$$

By Xiao's result [9], $C_{\Phi}: B^{\beta / 2} \longrightarrow B^{\beta / 2}$ is bounded. Furthermore, (16) yields 


$$
\frac{\left(1-|w|^{2}\right)^{\beta / 2}\left|\Phi^{\prime}(w)\right|}{\left(1-|\Phi(w)|^{2}\right)^{\beta / 2}} \leq C\left(1-|\Phi(w)|^{2}\right)^{(\alpha-\beta+2) / 2} \longrightarrow 0
$$

as $|\Phi(w)| \longrightarrow 1$. Thus, $C_{\Phi}: B^{\beta / 2} \longrightarrow B^{\beta / 2}$ is compact [9], and it follows as in [7] that $\|\Phi\|_{\infty}<1$. It has been established that the conditions $\Phi^{\prime} \in B^{\beta}, \Phi \Phi^{\prime} \in B^{\beta}$, and $\|\Phi\|_{\infty}<1$ are necessary if $D C_{\Phi}: F_{\alpha} \longrightarrow B^{\beta}$ is bounded.

Next, assume that $\Phi^{\prime} \in B^{\beta}, \Phi \Phi^{\prime} \in B^{\beta}$, and $\|\Phi\|_{\infty}<1$. To show that $D C_{\Phi}: F_{\alpha} \longrightarrow B^{\beta}$ is compact, let $\left(f_{n}\right)$ be a bounded sequence in $F_{\alpha}$ with $f_{n} \longrightarrow 0$ uniformly on compact subsets of $U$ as $n \longrightarrow \infty$. It is enough to prove that $\left\|D C_{\Phi}\left(f_{n}\right)\right\|_{B^{\beta}}$ $\longrightarrow 0$ as $n \longrightarrow \infty$. First, note that $\left|f_{n}{ }^{\prime}(\Phi(0)) \Phi^{\prime}(0)\right| \longrightarrow 0$ as $n \longrightarrow \infty$. For $z \in U$, (9) yields

$$
\begin{aligned}
\left(1-|z|^{2}\right)^{\beta}\left|\left(D C_{\Phi} f_{n}\right)^{\prime}(z)\right|= & \left(1-|z|^{2}\right)^{\beta} \mid f_{n}^{\prime \prime}(\Phi(z))\left(\Phi^{\prime}(z)\right)^{2} \\
& +f_{n}^{\prime}(\Phi(z)) \Phi^{\prime \prime}(z) \mid \\
\leq & C \max _{|w| \leq\|\Phi\|_{\infty}}\left|f_{n}^{\prime \prime}(w)\right| \\
& +\left\|\Phi^{\prime}\right\|_{B^{\beta}} \max _{|w| \leq\|\Phi\|_{\infty}}\left|f_{n}^{\prime}(w)\right| .
\end{aligned}
$$

Since $f_{n}{ }^{\prime} \longrightarrow 0$ and $f_{n}{ }^{\prime \prime} \longrightarrow 0$ uniformly on compact subsets as $n \longrightarrow \infty$, the argument shows that $\sup _{z \in U}\left(1-|z|^{2}\right)^{\beta}$ $\left(D C_{\Phi} f_{n}\right)^{\prime}(z) \mid \longrightarrow 0$ as $n \longrightarrow \infty$. Thus, $\left\|D C_{\Phi}\left(f_{n}\right)\right\|_{B^{\beta}} \longrightarrow 0$ as $n \longrightarrow \infty$, and $D C_{\Phi}: F_{\alpha} \longrightarrow B^{\beta}$ is compact, as required.

The remaining implication is clear, and the proof is complete.

Theorem 4. Fix $\alpha>0$ and $\beta \geq 2$. Let $\Phi$ be an analytic self-map of $U$. Then,

$$
\begin{aligned}
& D C_{\Phi}: F_{\alpha} \longrightarrow B^{\beta} \text { is bounded } \Leftrightarrow \\
& \sup _{z \in U} \frac{\left(1-|z|^{2}\right)^{\beta}\left|\Phi^{\prime \prime}(z)\right|}{\left(1-|\Phi(z)|^{2}\right)^{\alpha+1}}<\infty,
\end{aligned}
$$

and

$$
\sup _{z \in U} \frac{\left(1-|z|^{2}\right)^{\beta}\left|\Phi^{\prime}(z)\right|^{2}}{\left(1-|\Phi(z)|^{2}\right)^{\alpha+2}}<\infty
$$

Proof. Fix $\alpha, \beta$ and $\Phi$ as described.

First, assume (21) and (22). Let $f \in F_{\alpha}$. By (21) and the introductory remarks in Section 2,

$$
\begin{aligned}
\left(1-|z|^{2}\right)^{\beta}\left|f^{\prime}(\Phi(z))\right|\left|\Phi^{\prime \prime}(z)\right| & \leq\left(1-|z|^{2}\right)^{\beta}\left|\Phi^{\prime \prime}(z)\right| \frac{C\|f\|_{F_{\alpha}}}{\left(1-|\Phi(z)|^{2}\right)^{\alpha+1}} \\
& \leq C\|f\|_{F_{\alpha}} .
\end{aligned}
$$

A similar argument using (22) yields

$$
\left(1-|z|^{2}\right)^{\beta}\left|f^{\prime \prime}(\Phi(z))\right|\left|\Phi^{\prime}(z)\right|^{2} \leq C\|f\|_{F_{\alpha}}
$$

for all $z \in U$. Thus, $\sup _{z \in U}\left(1-|z|^{2}\right)^{\beta}\left|\left(D C_{\Phi} f\right)^{\prime}(z)\right| \leq C\|f\|_{F_{\alpha}}$. Since $\left|\left(D C_{\Phi} f\right)(0)\right| \leq C\|f\|_{F_{\alpha}}$, it now follows that $\left\|D C_{\Phi}(f)\right\|_{B^{\beta}} \leq C\|f\|_{F_{\alpha}}$, as required.

For the converse, assume that $\left\|D C_{\Phi}(f)\right\|_{B^{\beta}} \leq C\|f\|_{F_{\alpha}}$ for a constant $C$ independent of $f \in F_{\alpha}$. In particular, $\Phi^{\prime} \in B^{\beta}$.

The argument leading to (16) remains valid for $\beta \geq 2$. Thus, (22) holds. It remains to prove (21). First, note that

$$
\sup _{|\Phi(w)| \leq 1 / 2} \frac{\left(1-|w|^{2}\right)^{\beta}\left|\Phi^{\prime \prime}(w)\right|}{\left(1-|\Phi(w)|^{2}\right)^{\alpha+1}} \leq\left(\frac{4}{3}\right)^{\alpha+1}\left\|\Phi^{\prime}\right\|_{B^{\beta}}<\infty .
$$

For $w \in U$, define

$$
H_{w}(z)=\frac{(\alpha+3)\left(1-|\Phi(w)|^{2}\right)}{(1-\Phi \overline{(w) z})^{\alpha+1}}-\frac{(\alpha+1)\left(1-|\Phi(w)|^{2}\right)^{2}}{\left(1-\Phi \overline{(w) z)^{\alpha+2}}\right.}
$$

for $z \in U$. By Lemma 1 and Lemma 2, there is a constant $C$ independent of $w$ such that $\left\|H_{w}\right\|_{F_{\alpha}} \leq C$. Thus, $\left\|D C_{\Phi}\left(H_{w}\right)\right\|_{B^{\beta}} \leq C$ for all $w$. It follows that

$$
\sup _{z \in U}\left(1-|z|^{2}\right)^{\beta}\left|H_{w}{ }^{\prime}(\Phi(z)) \Phi^{\prime \prime}(z)+\left(\Phi^{\prime}(z)\right)^{2} H_{w}{ }^{\prime \prime}(\Phi(z))\right|<C,
$$

for all $w \in U$. An argument using $H_{w}{ }^{\prime}(\Phi(w))=(\alpha+1)$ $\Phi \overline{(w)} /\left(1-|\Phi(w)|^{2}\right)^{\alpha+1}$ and $H_{w}{ }^{\prime \prime}(\Phi(w))=0$ yields

$$
\sup _{1 / 2<|\Phi(w)|} \frac{\left(1-|w|^{2}\right)^{\beta}\left|\Phi^{\prime \prime}(w)\right|}{\left(1-|\Phi(w)|^{2}\right)^{\alpha+1}}<\infty
$$

The relations (25) and (28) establish relation (21), and the proof is complete.

Theorem 5. Fix $\alpha>0$ and assume $\beta \geq 2$. Let $\Phi$ be a self-map of $U$ for which $D C_{\Phi}: F_{\alpha} \longrightarrow B^{\beta}$ is bounded.

$$
D C_{\Phi}: F_{\alpha} \longrightarrow B^{\beta} \text { is compact } \Leftrightarrow
$$

$$
\lim _{|\Phi(z)| \longrightarrow 1} \frac{\left(1-|z|^{2}\right)^{\beta}\left|\Phi^{\prime \prime}(z)\right|}{\left(1-|\Phi(z)|^{2}\right)^{\alpha+1}}=0
$$

and

$$
\lim _{|\Phi(z)| \longrightarrow 1} \frac{\left(1-|z|^{2}\right)^{\beta}\left|\Phi^{\prime}(z)\right|^{2}}{\left(1-|\Phi(z)|^{2}\right)^{\alpha+2}}=0 .
$$


Proof. First, assume that $D C_{\Phi}: F_{\alpha} \longrightarrow B^{\beta}$ is bounded and relations (30) and (31) hold. Let $\left(f_{n}\right)$ be a bounded sequence in $F_{\alpha}$ such that $f_{n} \longrightarrow 0$ uniformly on compact subsets of $U$. As previously noted, there is a constant $C$ depending only on $\alpha$ such that

$$
\left(1-|z|^{2}\right)^{\beta}\left|f_{n}^{\prime}(\Phi(z))\right|\left|\Phi^{\prime}(z)\right|^{2} \leq C \frac{\left(1-|z|^{2}\right)^{\beta}\left|\Phi^{\prime}(z)\right|^{2}}{\left(1-|\Phi(z)|^{2}\right)^{\alpha+2}},
$$

for $n=1,2, \ldots$ and $z \in U$. Relation (31) now implies that given $\varepsilon>0$, there exists $r_{0}, 0<r_{0}<1$, such that

$$
\sup _{|\Phi(z)|>r_{0}}\left(1-|z|^{2}\right)^{\beta}\left|f_{n}{ }^{\prime \prime}(\Phi(z))\right|\left|\Phi^{\prime}(z)\right|^{2}<\varepsilon
$$

for all $n$.

Since $D C_{\Phi}: F_{\alpha} \longrightarrow B^{\beta}$ is bounded, relation (9) holds, and thus,

$$
\left(1-|z|^{2}\right)^{\beta}\left|f_{n}^{\prime}(\Phi(z))\right|\left|\Phi^{\prime}(z)\right|^{2}<C\left|f_{n}^{\prime \prime}(\Phi(z))\right|
$$

for all $z \in U$. Since $f_{n}{ }^{\prime \prime} \longrightarrow 0$ uniformly on $\left\{w:|w| \leq r_{0}\right\}$, there exists $N>0$ such that

$$
\sup _{|\Phi(z)| \leq r_{0}}\left(1-|z|^{2}\right)^{\beta}\left|f_{n}^{\prime \prime}(\Phi(z))\right|\left|\Phi^{\prime}(z)\right|^{2}<\varepsilon
$$

for all $n>N$. The relations (33) and (35) yield

$$
\sup _{z \in U}\left(1-|z|^{2}\right)^{\beta}\left|f_{n}^{\prime \prime}(\Phi(z))\right|\left|\Phi^{\prime}(z)\right|^{2}<\varepsilon,
$$

for $n>N$.

A similar argument using $\Phi^{\prime} \in B^{\beta}$ and (30) yields $N_{1}>0$ such that

$$
\sup _{z \in U}\left(1-|z|^{2}\right)^{\beta}\left|f_{n}^{\prime}(\Phi(z))\right|\left|\Phi^{\prime \prime}(z)\right|<\varepsilon
$$

for $n>N_{1}$. The relations (36) and (37) yield

$$
\sup _{z \in U}\left(1-|z|^{2}\right)^{\beta}\left|\left(D C_{\Phi} f_{n}\right)^{\prime}(z)\right| \longrightarrow 0
$$

as $n \longrightarrow \infty$.

Since $\left|\left(D C_{\Phi} f_{n}\right)(0)\right| \longrightarrow 0$ as $n \longrightarrow \infty$, the argument yields $\left\|D C_{\Phi}\left(f_{n}\right)\right\|_{B^{\beta}} \longrightarrow 0$ as $n \longrightarrow \infty$ for any sequence $\left(f_{n}\right)$ as described, and therefore, $D C_{\Phi}: F_{\alpha} \longrightarrow B^{\beta}$ is compact.

For the converse, assume that $D C_{\Phi}: F_{\alpha} \longrightarrow B^{\beta}$ is compact. We may assume that $\|\Phi\|_{\infty}=1$. Let $\left(z_{n}\right)$ be any sequence in $U$ with $\left|\Phi\left(z_{n}\right)\right| \longrightarrow 1$ as $n \longrightarrow \infty$. For $z \in U$, define

$$
f_{n}(z)=\frac{(\alpha+3)\left(1-\left|\Phi\left(z_{n}\right)\right|^{2}\right)}{\left(1-\Phi\left(z_{n}\right) z\right)^{\alpha+1}}-\frac{(\alpha+1)\left(1-\left|\Phi\left(z_{n}\right)\right|^{2}\right)^{2}}{\left(1-\Phi\left(z_{n}\right) z\right)^{\alpha+2}}
$$

By the lemmas above, $\left\|f_{n}\right\|_{F_{\alpha}} \leq C$. Also, $f_{n} \longrightarrow 0$ uniformly on compact subsets. Therefore, $\left\|D C_{\Phi}\left(f_{n}\right)\right\|_{B^{\beta}} \longrightarrow 0$ and

$$
\sup _{z \in U}\left(1-|z|^{2}\right)^{\beta}\left|f_{n}^{\prime}(\Phi(z)) \Phi^{\prime \prime}(z)+f_{n}^{\prime \prime}(\Phi(z))\left(\Phi^{\prime}(z)\right)^{2}\right| \longrightarrow 0
$$

as $n \longrightarrow \infty$. Calculations yield $f_{n}^{\prime \prime}\left(\Phi\left(z_{n}\right)\right)=0$ and

$$
f_{n}^{\prime}\left(\Phi\left(z_{n}\right)\right)=\frac{(\alpha+1) \Phi\left(\bar{z}_{n}\right)}{\left(1-\left|\Phi\left(z_{n}\right)\right|^{2}\right)^{\alpha+1}} .
$$

Substitution into (40) yields

$$
\frac{\left(1-\left|z_{n}\right|^{2}\right)^{\beta}\left|\Phi\left(z_{n}\right)\right|\left|\Phi^{\prime \prime}\left(z_{n}\right)\right|}{\left(1-\left|\Phi\left(z_{n}\right)\right|^{2}\right)^{\alpha+1}} \longrightarrow 0,
$$

as $n \longrightarrow \infty$. Since $\left(z_{n}\right)$ is a generic sequence with $\left|\Phi\left(z_{n}\right)\right| \longrightarrow 1$ as $n \longrightarrow \infty$, this yields the relation (30).

A similar argument using the functions

$$
g_{n}(z)=\frac{(\alpha+2)\left(1-\left|\Phi\left(z_{n}\right)\right|^{2}\right)}{\left(1-\Phi\left(\bar{z}_{n}\right) z\right)^{\alpha+1}}-\frac{(\alpha+1)\left(1-\left|\Phi\left(z_{n}\right)\right|^{2}\right)^{2}}{\left(1-\Phi\left(\bar{z}_{n}\right) z\right)^{\alpha+2}}
$$

yields the relation (31). The details are omitted.

Theorem 3 implies that if $D C_{\Phi}: F_{\alpha} \longrightarrow B^{\beta}$ is bounded for fixed $\alpha, \beta$ with $\beta<2$, then $D C_{\Phi}: F_{\gamma} \longrightarrow B^{\beta}$ is compact for all $\gamma>0$. The next corollary gives a related result when $\beta \geq 2$.

Corollary 6. Fix $\alpha>0$ and $\beta \geq 2$. Let $\Phi$ be a self-map of $U$ and assume that $D C_{\Phi}: F_{\alpha} \longrightarrow B^{\beta}$ is bounded. Then, $D C_{\Phi}: F_{\gamma}$ $\longrightarrow B^{\beta}$ is compact for any $\gamma, 0<\gamma<\alpha$.

Proof. By assumption, there is a constant $C$ such that $\left\|D C_{\Phi}(f)\right\|_{B^{\beta}} \leq C\|f\|_{F_{\alpha}}$ for all $f \in F_{\alpha}$. Fix $\gamma$ with $0<\gamma<\alpha$ and let $f \in F_{\gamma}$. Then, $f \in F_{\alpha}$ and $\|f\|_{F_{\alpha}} \leq\|f\|_{F_{\gamma}}$ [2]. Therefore, $D C_{\Phi}: F_{\gamma} \longrightarrow B^{\beta}$ is bounded and Theorem 5 applies.

Since $D C_{\Phi}: F_{\alpha} \longrightarrow B^{\beta}$ is bounded, (21) yields

$$
\frac{\left(1-|z|^{2}\right)^{\beta}\left|\Phi^{\prime \prime}(z)\right|}{\left(1-|\Phi(z)|^{2}\right)^{\gamma+1}} \leq C\left(1-|\Phi(z)|^{2}\right)^{\alpha-\gamma},
$$


and therefore,

$$
\lim _{|\Phi(z)| \rightarrow 1} \frac{\left(1-|z|^{2}\right)^{\beta}\left|\Phi^{\prime \prime}(z)\right|}{\left(1-|\Phi(z)|^{2}\right)^{\gamma+1}}=0 .
$$

A similar argument using (22) yields

$$
\lim _{|\Phi(z)| \rightarrow 1} \frac{\left(1-|z|^{2}\right)^{\beta}\left|\Phi^{\prime}(z)\right|^{2}}{\left(1-|\Phi(z)|^{2}\right)^{\gamma+2}}=0 .
$$

Theorem 5 now yields $D C_{\Phi}: F_{\gamma} \longrightarrow B^{\beta}$ is compact.

\section{The Operator $C_{\Phi} D$}

In this section, characterizations are given for self-maps $\Phi$ for which $C_{\Phi} D: F_{\alpha} \longrightarrow B^{\beta}$ is bounded or compact. The proofs are similar to those in Section 3, so details are kept to a minimum.

Theorem 7. Fix $\alpha>0$ and $0<\beta<1$.

$$
\begin{aligned}
C_{\Phi} D: F_{\alpha} \longrightarrow B^{\beta} \text { is bounded } \Leftrightarrow \\
\Phi \in B^{\beta} \text { and }\|\Phi\|_{\infty}<1 \Leftrightarrow \\
C_{\Phi} D: F_{\alpha} \longrightarrow B^{\beta} \text { is compact. }
\end{aligned}
$$

Proof. First, assume that there is a constant $C$ independent of $f \in F_{\alpha}$ such that $\left\|C_{\Phi} D(f)\right\|_{B^{\beta}} \leq C\|f\|_{F_{\alpha}}$. In particular, $\Phi \in B^{\beta}$ . For $w \in U$, define

$$
g_{w}(z)=\frac{1}{\left(1-\Phi \overline{(w) z)^{\alpha}}\right.}(z \in U) .
$$

There is a constant $C$ independent of $w \in U$ such that $\left\|g_{w}\right\|_{F_{\alpha}} \leq C$, and it follows that

$$
\sup _{z \in U}\left(1-|z|^{2}\right)^{\beta}\left|g_{w}{ }^{\prime \prime}(\Phi(z)) \Phi^{\prime}(z)\right|<C,
$$

for all $w \in U$. The substitution $z=w$ yields

$$
\left(1-|w|^{2}\right)^{\beta} \frac{\alpha(\alpha+1)|\Phi(w)|^{2}\left|\Phi^{\prime}(w)\right|}{\left(1-|\Phi(w)|^{2}\right)^{\alpha+2}}<C,
$$

for all $w \in U$. Therefore,

$$
\sup _{1 / 2<|\Phi(w)|} \frac{\left(1-|w|^{2}\right)^{\beta}\left|\Phi^{\prime}(w)\right|}{\left(1-|\Phi(w)|^{2}\right)^{\alpha+2}}<\infty .
$$

Since $\Phi \in B^{\beta}$,

$$
\sup _{|\Phi(w)| \leq 1 / 2} \frac{\left(1-|w|^{2}\right)^{\beta}\left|\Phi^{\prime}(w)\right|}{\left(1-|\Phi(w)|^{2}\right)^{\alpha+2}}<\infty .
$$

It follows that

$$
\sup _{w \in U} \frac{\left(1-|w|^{2}\right)^{\beta}\left|\Phi^{\prime}(w)\right|}{\left(1-|\Phi(w)|^{2}\right)^{\alpha+2}}<\infty
$$

and therefore

$$
\sup _{w \in U} \frac{\left(1-|w|^{2}\right)^{\beta}\left|\Phi^{\prime}(w)\right|}{\left(1-|\Phi(w)|^{2}\right)^{\beta}}<\infty .
$$

By [9], $C_{\Phi}: B^{\beta} \longrightarrow B^{\beta}$ is bounded. A further argument as in the proof of Theorem 3 yields that $C_{\Phi}: B^{\beta} \longrightarrow B^{\beta}$ is compact. Since $\beta<1$, Shapiro's result [7] applies and yields $\|\Phi\|_{\infty}<1$. Thus, the conditions $\Phi \in B^{\beta}$ and $\|\Phi\|_{\infty}<1$ are necessary in order for $C_{\Phi} D: F_{\alpha} \longrightarrow B^{\beta}$ to be bounded.

Next, assume $\Phi \in B^{\beta}$ and $\|\Phi\|_{\infty}<1$. Let $\left(f_{n}\right)$ be a bounded sequence in $F_{\alpha}$ with $f_{n} \longrightarrow 0$ uniformly on compact subsets of $U$. First, note that $\left|f_{n}{ }^{\prime}(\Phi(0))\right| \longrightarrow 0$ as $n \longrightarrow \infty$. For $z \in U$,

$$
\left(1-|z|^{2}\right)^{\beta}\left|\left(f_{n}{ }^{\prime} \circ \Phi\right)^{\prime}(z)\right| \leq\|\Phi\|_{B^{\beta}} \max _{|w| \leq\|\Phi\|_{\infty}}\left|f_{n}{ }^{\prime \prime}(w)\right| .
$$

Since $f_{n}{ }^{\prime \prime} \longrightarrow 0$ uniformly on compact subsets, the argument yields $\left\|C_{\Phi} D\left(f_{n}\right)\right\|_{B^{\beta}} \longrightarrow 0$ and $C_{\Phi} D: F_{\alpha} \longrightarrow B^{\beta}$ is compact.

The remaining implication is trivial, and the proof is complete.

Theorem 8. Fix $\alpha>0$ and $\beta \geq 1$. Let $\Phi$ be a self-map of $U$.

$$
\begin{aligned}
C_{\Phi} D: & F_{\alpha} \longrightarrow B^{\beta} \text { is bounded } \Leftrightarrow \\
& \sup _{z \in U} \frac{\left(1-|z|^{2}\right)^{\beta}\left|\Phi^{\prime}(z)\right|}{\left(1-|\Phi(z)|^{2}\right)^{\alpha+2}}<\infty .
\end{aligned}
$$

Proof. First, assume that the supremum is finite.

Let $f \in F_{\alpha}$. By previous remarks, $\left|f^{\prime}(\Phi(0))\right| \leq C\|f\|_{F_{\alpha}}$. By an argument as in the proof of Theorem 4,

$$
\begin{aligned}
\left(1-|z|^{2}\right)^{\beta}\left|\left(f^{\prime} \circ \Phi\right)^{\prime}(z)\right| & =\left(1-|z|^{2}\right)^{\beta}\left|f^{\prime \prime}(\Phi(z))\right|\left|\Phi^{\prime}(z)\right| \\
& \leq\left(1-|z|^{2}\right)^{\beta} \frac{C\|f\|_{F_{\alpha}}\left|\Phi^{\prime}(z)\right|}{\left(1-|\Phi(z)|^{2}\right)^{\alpha+2}} \\
& \leq C\|f\|_{F_{\alpha}},
\end{aligned}
$$

and thus, $\left\|C_{\Phi} D(f)\right\|_{B^{\beta}} \leq C\|f\|_{F_{\alpha}}$ as required.

To complete the proof, assume that $\left\|C_{\Phi} D(f)\right\|_{B^{\beta}} \leq C$ $\|f\|_{F_{\alpha}}$ for a constant $C$ independent of $f$. The argument leading to (53) remains valid for $\beta \geq 1$. This proves the opposite implication, and the proof is complete. 
Theorem 9. Fix $\alpha>0$ and $\beta \geq 1$. Let $\Phi$ be a self-map of $U$ and assume that $C_{\Phi} D: F_{\alpha} \longrightarrow B^{\beta}$ is bounded.

$$
\begin{aligned}
C_{\Phi} D: & F_{\alpha} \longrightarrow B^{\beta} \text { is compact } \Leftrightarrow \\
& \lim _{|\Phi(z)| \longrightarrow 1} \frac{\left(1-|z|^{2}\right)^{\beta}\left|\Phi^{\prime}(z)\right|}{\left(1-|\Phi(z)|^{2}\right)^{\alpha+2}}=0 .
\end{aligned}
$$

Proof. First, assume that $C_{\Phi} D: F_{\alpha} \longrightarrow B^{\beta}$ is bounded and the limit condition holds. Let $\left(f_{n}\right)$ be a bounded sequence in $F_{\alpha}$ with $f_{n} \longrightarrow 0$ uniformly on compact subsets as $n \longrightarrow$ $\infty$. Clearly, $\left|f_{n}{ }^{\prime}(\Phi(0))\right| \longrightarrow 0$ as $n \longrightarrow \infty$. As in previous arguments,

$$
\left(1-|z|^{2}\right)^{\beta}\left|f_{n}^{\prime \prime}(\Phi(z))\right|\left|\Phi^{\prime}(z)\right| \leq C \frac{\left(1-|z|^{2}\right)^{\beta}\left|\Phi^{\prime}(z)\right|}{\left(1-|\Phi(z)|^{2}\right)^{\alpha+2}},
$$

for all $z \in U$. The hypothesis now implies that, given $\varepsilon>0$, there exists $r_{0}, 0<r_{0}<1$, such that

$$
\sup _{|\Phi(z)|>r_{0}}\left(1-|z|^{2}\right)^{\beta}\left|f_{n}^{\prime \prime}(\Phi(z))\right|\left|\Phi^{\prime}(z)\right|<\varepsilon
$$

for all $n$. Since $\Phi \in B^{\beta}$ and since $f_{n}{ }^{\prime \prime} \longrightarrow 0$ uniformly on compact subsets,

$$
\sup _{|\Phi(z)| \leq r_{0}}\left(1-|z|^{2}\right)^{\beta}\left|f_{n}{ }^{\prime \prime}(\Phi(z))\right|\left|\Phi^{\prime}(z)\right| \longrightarrow 0,
$$

as $n \longrightarrow \infty$. By (60) and (61),

$$
\sup _{z \in U}\left(1-|z|^{2}\right)^{\beta}\left|\left(f_{n}{ }^{\prime} \circ \Phi\right)^{\prime}(z)\right| \longrightarrow 0,
$$

as $n \longrightarrow \infty$. The argument yields $\left\|f_{n}{ }^{\prime} \circ \Phi\right\|_{B^{\beta}} \longrightarrow 0$ as $n \longrightarrow \infty$ for any sequence $\left(f_{n}\right)$ as described above. Thus, $C_{\Phi} D: F_{\alpha} \longrightarrow B^{\beta}$ is compact.

Now, assume that $C_{\Phi} D: F_{\alpha} \longrightarrow B^{\beta}$ is compact. We may assume that $\|\Phi\|_{\infty}=1$. Let $\left(z_{n}\right)$ be any sequence in $U$ with $\left|\Phi\left(z_{n}\right)\right| \longrightarrow 1$ as $n \longrightarrow \infty$. For $n=1,2, \cdots$, define

$$
f_{n}(z)=\frac{1-\left|\Phi\left(z_{n}\right)\right|^{2}}{\left(1-\Phi\left(\bar{z}_{n}\right) z\right)^{\alpha+1}}
$$

for $z \in U$. By Lemma $1,\left\|f_{n}\right\|_{F_{\alpha}} \leq C$ for all $n$. Also, $f_{n} \longrightarrow 0$ uniformly on compact subsets. Therefore, $\left\|C_{\Phi} D\left(f_{n}\right)\right\|_{B^{\beta}}$ $\longrightarrow 0$ as $n \longrightarrow \infty$. Given $\varepsilon>0$, there exists $N>0$ such that

$$
\sup _{z \in U}\left(1-|z|^{2}\right)^{\beta}\left|f_{n}^{\prime \prime}(\Phi(z))\right|\left|\Phi^{\prime}(z)\right|<\varepsilon,
$$

for all $n>N$. In particular, $\left(1-\left|z_{n}\right|^{2}\right)^{\beta}\left|f_{n}{ }^{\prime \prime}\left(\Phi\left(z_{n}\right)\right)\right|\left|\Phi^{\prime}\left(z_{n}\right)\right|$ $<\varepsilon$ for $n>N$. Calculations yield

$$
\frac{\left(1-\left|z_{n}\right|^{2}\right)^{\beta}(\alpha+1)(\alpha+2)\left|\Phi\left(z_{n}\right)\right|^{2}\left|\Phi^{\prime}\left(z_{n}\right)\right|}{\left(1-\left|\Phi\left(z_{n}\right)\right|^{2}\right)^{\alpha+2}}<\varepsilon
$$

for $n>N$. Since $\left(z_{n}\right)$ is a generic sequence with $\left|\Phi\left(z_{n}\right)\right| \longrightarrow 1$, it follows that

$$
\lim _{|\Phi(z)| \longrightarrow 1} \frac{\left(1-|z|^{2}\right)^{\beta}\left|\Phi^{\prime}(z)\right|}{\left(1-|\Phi(z)|^{2}\right)^{\alpha+2}}=0 .
$$

The proof is complete.

Assume that $C_{\Phi} D: F_{\alpha} \longrightarrow B^{\beta}$ is bounded for fixed $\alpha>0$ and $\beta<1$. By Theorem 7, $\Phi \in B^{\beta}$ and $\|\Phi\|_{\infty}<1$. It follows that $C_{\Phi} D: F_{\gamma} \longrightarrow B^{\beta}$ is compact for any $\gamma>0$. Corollary 10 gives a related result in the case $\beta \geq 1$.

Corollary 10. Fix $\alpha>0, \beta \geq 1$ and assume that $C_{\Phi} D: F_{\alpha}$ $\longrightarrow B^{\beta}$ is bounded. Then, $C_{\Phi} D: F_{\gamma} \longrightarrow B^{\beta}$ is compact for any $\gamma, 0<\gamma<\alpha$.

Proof. Fix $0<\gamma<\alpha$ and let $f \in F_{\gamma}$. Then, $f \in F_{\alpha}$ and $\|f\|_{F_{\alpha}}$ $\leq\|f\|_{F_{\gamma}}[2]$. Therefore, $C_{\Phi} D: F_{\gamma} \longrightarrow B^{\beta}$ is bounded and Theorem 9 applies.

By Theorem 8 , there is a constant $C$ with

$$
\frac{\left(1-|z|^{2}\right)^{\beta}\left|\Phi^{\prime}(z)\right|}{\left(1-|\Phi(z)|^{2}\right)^{\alpha+2}} \leq C,
$$

for all $z \in U$. Therefore,

$$
\frac{\left(1-|z|^{2}\right)^{\beta}\left|\Phi^{\prime}(z)\right|}{\left(1-|\Phi(z)|^{2}\right)^{\gamma+2}} \leq C\left(1-|\Phi(z)|^{2}\right)^{\alpha-\gamma} \longrightarrow 0,
$$

as $|\Phi(z)| \longrightarrow 1$. By Theorem 9, $C_{\Phi} D: F_{\gamma} \longrightarrow B^{\beta}$ is compact.

\section{Data Availability}

This manuscript does not contain any data.

\section{Conflicts of Interest}

The author declares that there is no conflict of interest regarding the publication of this paper.

\section{Acknowledgments}

This research was conducted under the employ of the Department of Mathematics and Statistics at the University of New Hampshire, Durham, NH, USA. 


\section{References}

[1] J. A. Cima, A. L. Matheson, and W. T. Ross, The Cauchy Transform, Mathematical Surveys and Monographs, vol. 125, American Mathematical Society, Providence, 2006.

[2] R. A. Hibschweiler and T. H. Mac Gregor, Fractional Cauchy Transforms, Chapman and Hall/CRC, Boca Raton, 2006.

[3] S. Ohno, "Products of differentiation and composition on Bloch spaces," Bulletin of the Korean Mathematical Society, vol. 46, no. 6, pp. 1135-1140, 2009.

[4] S. Li and S. Stević, "Composition followed by differentiation between the weighted Bergman spaces and Bloch type spaces," Journal of Applied Functional Analysis, vol. 3, no. 3, pp. 333340, 2008.

[5] R. A. Hibschweiler and N. Portnoy, "Composition followed by differentiation between Bergman and Hardy spaces," Rocky Mountain Journal of Mathematics, vol. 35, pp. 843-866, 2005.

[6] R. A. Hibschweiler, "Weighted composition operators between the fractional Cauchy spaces and the Bloch-type spaces," Journal of Complex Analysis, vol. 2017, Article ID 9486907, 5 pages, 2017.

[7] J. H. Shapiro, "Compact composition operators on spaces of boundary-regular holomorphic functions," Proceedings of the American Mathematical Society, vol. 100, no. 1, pp. 49-57, 1987.

[8] C. Cowen and B. D. Mac Cluer, Composition Operators on Spaces of Analytic Functions, CRC Press, Boca Raton, 1995.

[9] J. Xiao, "Composition operators associated with Bloch-type spaces," Complex Variables and Elliptic Equations, vol. 46, pp. 109-121, 2001. 